\title{
MODELING ROLLERS USING TERRESTRIAL LIDAR POINTS IN A HOT-ROLLING STEEL MILL
}

\author{
Sagar S Deshpande ${ }^{a}$, Mike Falk ${ }^{b}$, Nate Plooster ${ }^{b}$ \\ ${ }^{a}$ Surveying Engineering, Penn State University, USA, ssd5269@psu.edu, ${ }^{b}$ Falk-PLI, Portage, Indiana, USA, mfalk@ falk-pli.com
}

Commission VI, WG VI/4

KEY WORDS: Hot rollling steel-mill, Rollers, terrestrial lidar

\begin{abstract}
:
Terrestrial lidar scanners are increasingly being used in numerous indoor mapping applications. This paper presents a methodology to model rollers used in hot-rolling steel mills. Hot-rolling steel mills are large facilities where steel is processed to different shapes. In a steel sheet manufacturing process, a steel slab is reheated at one end of the mill and is passed through multiple presses to achieve the desired cross-section. Hundreds of steel rollers are used to transport the steel slab from one end of the mill to the other. Over a period of use, these rollers wore out and need replacement. Manual determination of the damage to the rollers is a time-consuming task. Moreover, manual measurements can be influenced by the operator's judgment. This paper presents a methodology to model rollers in a hot-rolling steel mill using lidar points. A terrestrial lidar scanner was used to collect lidar points over the roller surfaces. Data from several stations were merged to create a single point cloud. Using a bounding box, lidar points on all the rollers were clipped and used in this paper. The clipped data consisted of the roller as well as outlier points. Depending on the scan angles of scanner stations, partial surfaces of the rollers were scanned. A right-handed coordinate frame was used where the X-axis passed through the centers of all the rollers, Y-axis was parallel to the length of the first roller, and the Z-axis was in the plumb direction. Using a standard diameter of the roller, model roller points were created to extract the rollers. Both the lidar data and the model points were converted to rectangular prism-shaped voxels of dimensions $15.24 \mathrm{~mm}(0.05 \mathrm{ft}) \mathrm{x} 15.24 \mathrm{~mm}$ in the $\mathrm{X}$ and $\mathrm{Z}$ directions and extending over the entire width of the roller in the Y-direction. Voxels containing at least 40 lidar points were considered valid. Binary images of both the lidar points and the model points were created in the $\mathrm{X}-\mathrm{Z}$ axes using the valid voxels. The roller locations in the lidar image were located by performing 2D FFT image matching using the model roller image. The roller points at the shortlisted locations were fitted with a circle equation to determine the mean roller diameters and mean center locations (roller's rotation axis). The outlier points were filtered in this process for each roller. The elevation at the top of every roller was determined by adding their radii and Z-coordinates of its centers. Incorrectly located and/or modeled rollers were identified by implementing moving-average filters. Positively identified roller points were further analyzed to determine surface erosions and tilts. The above methodology showed that the rollers can be effectively modeled using the lidar points.
\end{abstract}

\section{INTRODUCTION}

Hot-rolling steel mills are large facilities where steel is processed into different shapes. Figure 1 shows a schematic diagram of a steel sheet manufacturing hot-rolling mill. The steelmanufacturing process starts with a cold slab at one end that is reheated in a furnace and then passed through multiple mills to achieve the desired thickness and cross-section. In this process, rollers are placed at regular intervals to transport the slab. The length over which a slab is processed in a steel mill can extend to six to nine hundred meters. In the end, the processed steel sheet is rolled to form a coil.

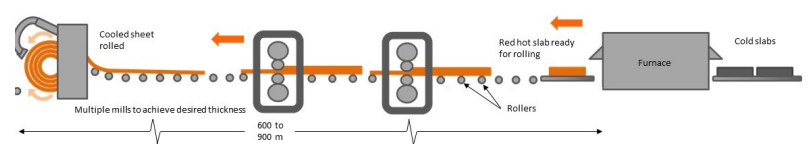

Figure 1. Schematic diagram showing the working of a hot-rolling steel mill.

Figure 2 shows rollers in a hot-rolling steel mill. Drive motors are attached to one end of rollers that spin them at a constant rotational speed.

Over a period, the rollers undergo wear and tear because of friction with the slabs. They could develop several issues such as uneven surface profile and tilt in the rotation axis as shown in Figure 3. The unevenness, although a few millimeters, can affect the quality of the steel by creating differential roller surface speed which causes stripes on the steel surface. As shown in Figure 3(a), the axis of a roller may not be perfectly horizontal. Such a roller, due to varying friction over its length, could result in different roller radii. Figure 3(b) shows a roller with changing radii along its length.

Figure 4 shows changes in diameters from roller to roller. As stated earlier, the rollers are rotated at a constant rate. Uneven reductions in radii along the length of rollers would result in uneven surface velocities causing friction and/or drag between the rollers and steel (PI tapes). Figure 4 also shows that the change in the radius of the rollers over their length could result in an uneven top surface. This situation is shown by the third roller where a steel section could be supported by the second and fourth rollers. The third roller might just touch or scratch the steel over it.

Thus, it can be concluded that the quality of the steel produced directly depends on the surface quality of these rollers. Until now, the surface variations and alignments of the rollers have been measured manually using tactile direct measurement tools. The mill operations had to be stopped to make manual measurements thereby causing loss of revenue for the steel factory. 


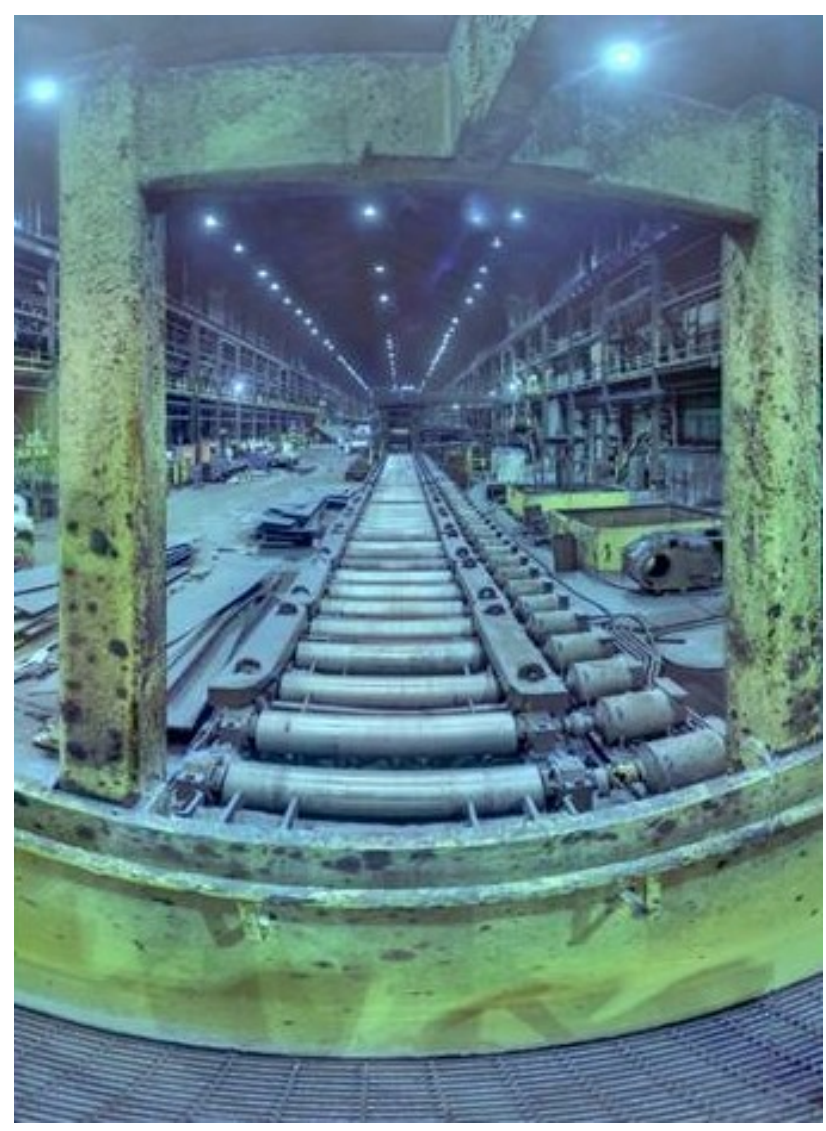

Figure 2. Rollers in a steel mill. Drive motors on the right side of the rollers.

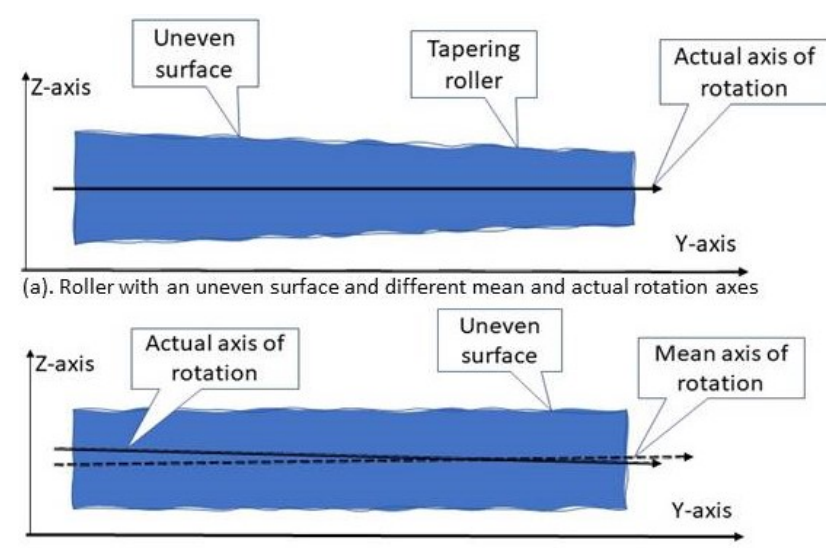

(b). Roller with uneven tapering surface and actual rotation axis

Figure 3. Cross-sectional view of rollers showing surface erosion, mean and tilted rotational axes.

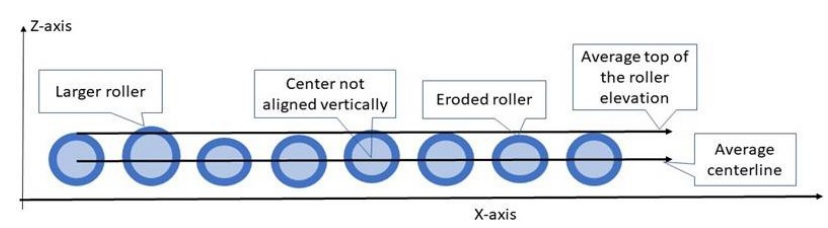

Figure 4. Issues in multiple rollers due to uneven erosion.

Moreover, such measurements can be made at limited locations. Hence, more time and efforts would be required to manually measure the entire profile of the rollers.
Alternatively, a laser scanner can be employed to scan the rollers. But manual extraction of the important roller parameters from the laser points would be highly time-consuming and would be influenced by individual operator judgment which could lead to errors from misinterpretation. Hence, methods that can automatically extract the roller features and minimize manual interaction can greatly increase the effectiveness of roller mapping.

In this paper, methods are presented to extract the roller parameters from terrestrial laser points. The main objectives are to minimize human factor error, increase the measurement level of confidence, and to make the process computationally efficient.

\section{LITERATURE REVIEW}

Laser scanners (Light Detection and Ranging (lidar)) are increasingly being used on aerial or terrestrial platforms. The basic principle of a laser scanner is based on distance measurement using a laser pulse sent by a sensor to the object (Liu et al., 2009). An onboard GPS/IMU system coupled with the range distance can determine the position of the ground point. Due to its direct ranging capabilities and few limitations, lidar is increasingly being used in numerous industries with applications such as structural shape modeling (Puente et al., 2016, Yi et al., 2019), geometric deformation modeling (Cabaleiro et al., 2015, Selvaraj and Madhavan, 2018), deformation determination in structural members (Cabaleiro et al., 2014), transportation engineering (Gézero and Antunes, 2019), and water resources engineering (Deshpande, 2013, Deshpande and Yilmaz, 2017). The paper (Taheri Andani et al., 2018) showed the reliability of lidar sensors for the assessment of the top of rail lubricity in a laboratory environment. The paper (Stein et al., 2016) presented methods to determine infrastructure elements like rails and turnout from mobile lidar data of a railroad. The paper (Cabaleiro et al., 2016) presented an application of lidar technology in structural health monitoring, especially in the study of deformations and stresses in beams. In the paper, (Gézero and Antunes, 2019) 3D linear elements were extracted from a mobile lidar point cloud of a railroad. In the papers, (Sánchez-Rodríguez et al., 2019, Yi et al., 2019, Puente et al., 2016) methodologies addressing different issues in automatic tunnel modeling were presented.

Thus, it could be summarized based on the literature review that lidar is progressively used for modeling and mapping various construction elements. However, the use of laser scanners in steel industries is relatively new. Steel mills have numerous structural elements that need periodic monitoring for the smooth functioning of their facilities and the safety of their employees. Rollers, one of the important elements in the steel-mill industry are cylindrical in shape. Commercially available software cannot address the issues to the extent necessary in steel mills. This paper presented unique roller modeling approaches using a terrestrial laser scanner in steel mills.

\subsection{Salient metric properties of a roller in a steel mill}

As stated earlier, the rollers are an essential component in steel mills. In this section, different issues that are being addressed by this paper are listed. Considering all rollers together (Figure 4) following pertinent parameters were determined:

1. Centerline variations of rollers (Axial 3D alignment)

2. Radius variations of the rollers (Roller profile/wear) 


\section{High point elevations of rollers}

Pertinent parameters considering an individual roller (Figure 3)

1. Surface variations of a roller with reference to the mean rotation axis

2. The actual (tilted) rotation axis variation compared to the mean rotation axis

3. Surface variations considering the roller's actual (tilted) rotation axis

\section{DATA DESCRIPTION}

The data used in this study was acquired in a steel mill located in the US continent using a Z+F IMAGER $\AA$ 5010C, 3D Laser Scanner. The rollers were scanned by stationing the instrument on bridges as shown in Figure 2. Lidar points on 372 rollers were clipped by defining a bounding box. It can be noted from Figure 5 that several outliers existed in the clipped dataset. A right-handed local coordinate system was used to orient the data in which the $\mathrm{X}$-axis was along the centerline of the rollers and the $\mathrm{Z}$-axis was in the plumb direction.

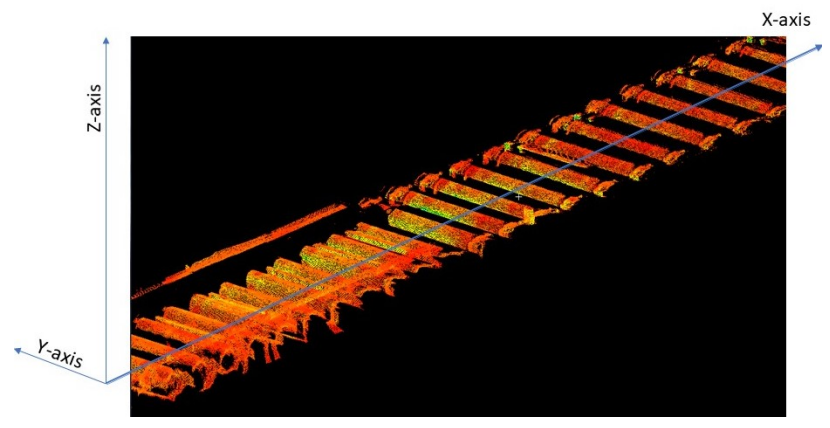

Figure 5. Point cloud of the rollers in a steel mill and the coordinate reference frame.

The roller points and the outlier points within a sub-set of five rollers are shown in Figure 6(a). It can be noted that depending on the locations of the scanners and their scan angles, laser points were collected on limited surfaces of rollers.

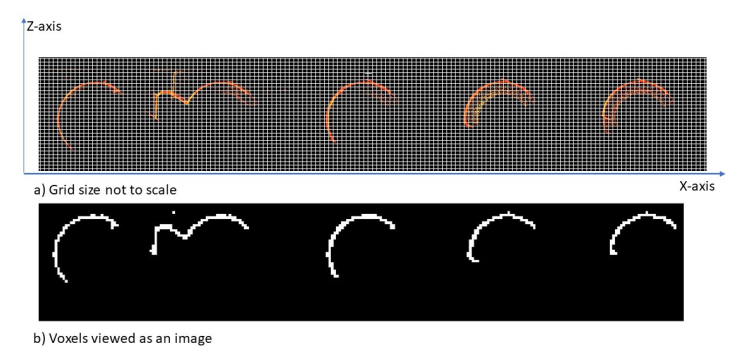

Figure 6. Laser points in the X-Z plane. (a) Voxel overlaid on the point cloud and (b) image created by thresholding the number of laser points within each voxel.

\section{METHODOLOGY}

The overview of the entire process is shown in Figure 7 which can be broadly grouped as locating the rollers using 2D Fast
Fourier Transform (FFT) and extracting rollers' metric parameters.

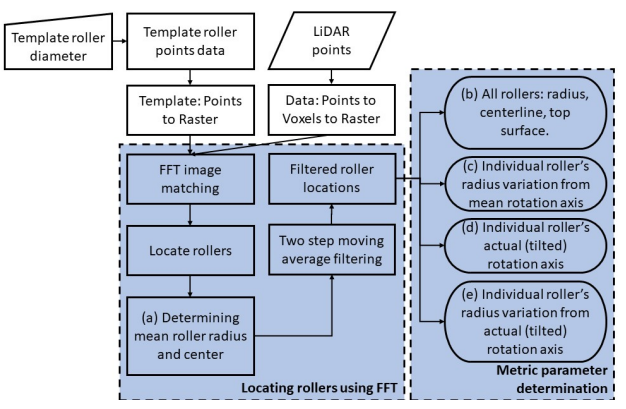

Figure 7. Steps to extract roller properties.

\subsection{Two-dimensional Fast Fourier Transform (2D FFT) im- age matching}

The general idea of 2D Discrete Fourier Transform is that an image in the spatial domain $\mathrm{f}(\mathrm{x}, \mathrm{y})$ of size $(\mathrm{M}$ by $\mathrm{N})$ pixels will be represented in the frequency domain $\mathrm{F}(\mathrm{u}, \mathrm{v})$ (Smith, 1997, Gonzalez et al., 2004). Figure 8 shows a digital image in the spatial and the frequency domains, respectively. The concept behind the Fourier transform is that any waveform can be constructed using a sum of sine and cosine waves of different frequencies.

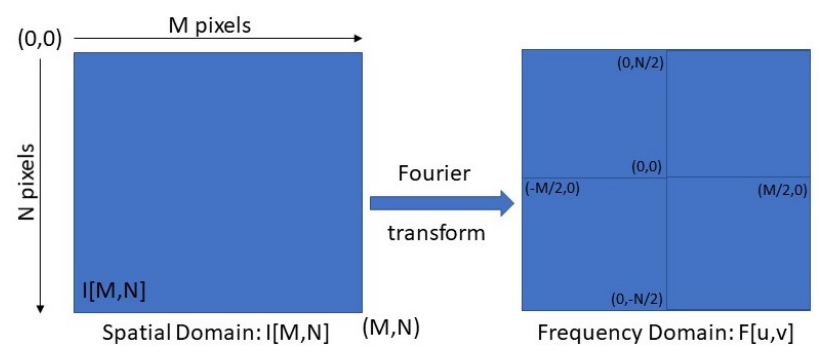

Figure 8. 2D FFT.

Thus, $\mathrm{F}(\mathrm{u}, \mathrm{v})$ can be converted to the corresponding image ( $\mathrm{f}(\mathrm{x}, \mathrm{y}))$ using the Inverse Fourier transform (IFT). Fast Fourier transform (FFT) is a smart algorithm for rapidly calculating the DFT. FFT can perform convolution by multiplication in the frequency domain hundreds of times faster than convolution in spatial domain. Using this concept, two images can be convoluted in the frequency domain for image matching (Smith et al., 1997). This process is faster than the correlation in the spatial domain.

Figure 9(a) shows a search template that is to be located on the input image. According to the FFT matching, the template image is flipped from left to right and then from top to bottom. A DFT is applied to both the images to obtain the real and imaginary output. These two sets are multiplied to obtain a real and imaginary output. An IFT transform is implemented to obtain the correlation. The rightmost image in Figure 9(b) shows the results, where the brighter pixels represent the locations of higher correlation. This technique is used to locate the rollers to expedite the matching process.

\subsection{Locating the rollers using FFT matching}

Figure 5 shows the point cloud and the coordinate reference frame. To locate the rollers, the entire laser point cloud was 

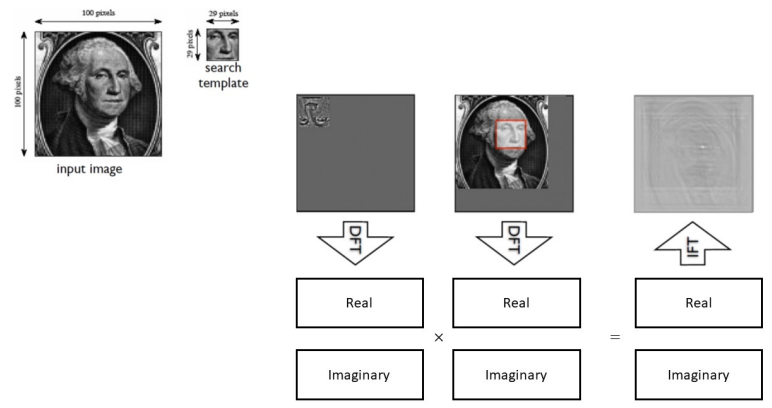

Figure 9. FFT image matching.

converted to voxels. Voxels are 3D cubes represented by width, depth, and height in $\mathrm{X}, \mathrm{Y}$, and $\mathrm{Z}$ directions, respectively. Several researchers have used voxels in various LiDAR-based applications (Popescu and Zhao, 2008, Chasmer et al., 2004).

In this study, rectangular prism-shaped voxels of dimensions $15.24 \mathrm{~mm}(0.05 \mathrm{ft}) \times 15.24 \mathrm{~mm}$ in the $\mathrm{X}$ and $\mathrm{Z}$ directions and extending over the entire width in the Y-direction were created. It can be noted from Figure 6(a) that the voxels would represent a $2 \mathrm{D}$ image in which the column and row directions were aligned to the $\mathrm{X}$ and $\mathrm{Z}$ axes of the data, respectively. The first column of the raster image was placed on the point with the smallest $\mathrm{X}$ value and the first row was placed on the point with the highest $\mathrm{Z}$ value. Voxels containing at least forty points were considered valid. This threshold was based on experimental testing which showed a reduction in outlier cells in the raster image. A binary image was created along the $\mathrm{X}$ and $\mathrm{Z}$ axis containing 1 for a valid voxel and 0 for a non-valid voxel. Figure $6(\mathrm{~b})$ shows the resulting image where white shows the valid pixel containing at least forty laser points. The shape of the roller and several outlier pixels can be seen in this figure. In the next step, individual rollers were located using the FFT image matching technique where Figure 6(b) was used as the input image.

In a steel mill, a standard roller size is used. Therefore, depending on the source of the data, a template roller was created by specifying the standard roller radius of $20.7 \mathrm{~cm}(0.68 \mathrm{ft})$. Virtual points were placed at the specified radius (Figure 10(a)) and were grouped in voxels of size $15.24 \mathrm{~mm}(0.05 \mathrm{ft}) \times 15.24 \mathrm{~mm}$ in the $\mathrm{X}$ and $\mathrm{Z}$ directions. The voxels were converted to create a template search image as shown in Figure 10(b).
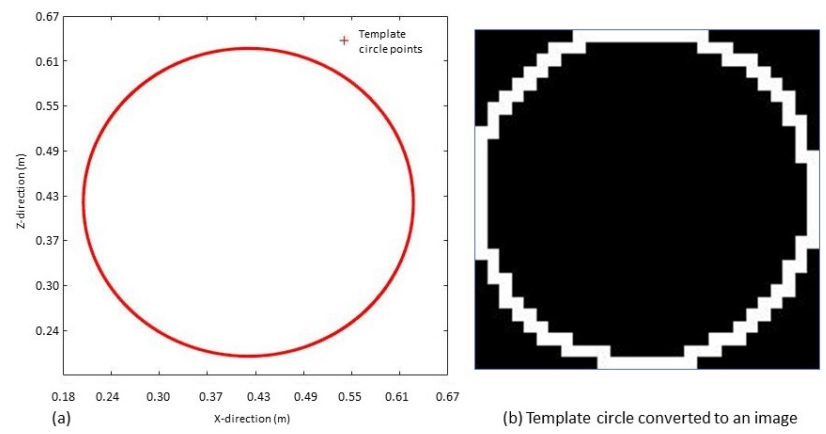

Figure 10. FFT image matching (Smith (1997)).

This template image was used for FFT image matching to locate the rollers in the input image. The resulting correlation image is shown in Figure 11(a). It should be noted that the template was a complete circle whereas the rollers were partial circles with varying surface point coverage. Depending on the extent of roller surface points, the correlation value ranged significantly over the entire length. However, as seen in Figure 6(a), the extents of rollers' surface scan changed gradually in a smaller region. Hence, to obtain a uniform correlation, the entire length was divided into smaller sections along the X-direction, so that a local threshold could be determined. Approximately five rollers were processed at one time thereby generating a uniform correlation value for every section. Sufficient overlap was maintained between these sections to avoid missing any roller at their ends. Based on multiple testing a threshold of Max value/2 was adopted for each section to locate the rollers. Here Max value represented the maximum correlation value within each section. Figure 11(b) shows a binary image where white pixels represented locations with values greater than Max value/2 . It can be noticed that a cluster of white pixels existed at the roller locations. At this point, user input was used to specify a seed point as shown in the figure. Rollers were searched within a buffer of three pixels on both sides along the row of the seed pixel. When a cluster was detected, their average location in row and column directions was considered as a matching location. It should be noted that FFT matching located the lower right corner of the rollers. Using this location, the left and right extents in the $\mathrm{X}$-direction and top and bottom in the Z-direction for every roller on the image were identified as below:

Left extent=Center column pixel-size of template circle (DIA)4 pixels (OFFSET)

Right extent=Center column pixel+4 pixels $($ OFFSET)

Top extent=Center row pixel-size of template circle (DIA)-4 pixels (OFFSET)

Bottom extent=Center row pixel+4 pixels $($ OFFSET)

These measurements are shown in Figure 11(b) where DIA stands for the diameter of the template circle and OFFSET stands for four pixels offset. The OFFSET pixels accounted for rollers' size variations. Figure 11(c) shows the extents of the identified rollers. Laser points on every roller were identified using the extents determined. At this stage, the locations of every roller were available. Using the points within these extents, various parameters were extracted as described below.

4.2.1 Determining the mean radius and mean rotation axis of individual roller: The points within the extents of a roller consisted of outliers and roller points. Therefore, it was necessary to exclude the outliers to determine the average radius and center of the roller. An iterative refinement approach was implemented to determine the actual radius and the center of the roller as shown in Figure 12. A threshold of $6.1 \mathrm{~mm}(0.02 \mathrm{ft}$ $-1 / 8$ th of an inch) was used after several tests to shortlist the points belonging to the roller. Points within this threshold were used to fit a circle, compute the radius, and coordinates of the center of the fitted circle.

Figure 13 shows laser points within the extents of one roller containing the filtered roller points, outlier points, and the fitted circle. The coordinates of the center were considered as the mean axis of rotation for the roller.

It was found that the above methodology erroneously selected a few incorrect roller locations. Based on visual examination, it was found that the erroneously detected rollers had significantly 


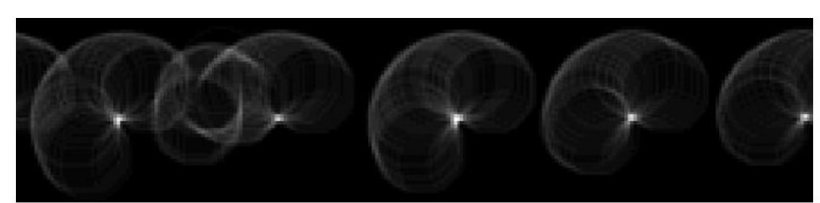

(a) FFT matching result. Brighter pixels represent matches. Range from 0 to 41.

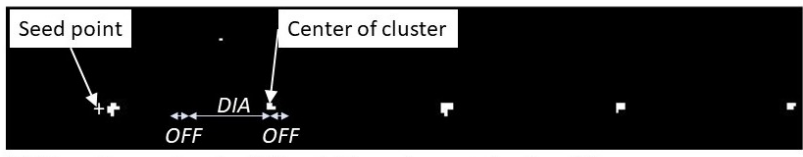

(b) Binary image showing FFT matching values greater than 20.

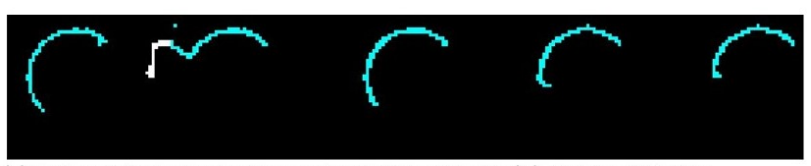

(c) Rollers detected using the seed point identified in (b). Points within the temnlate circle size selected.

Figure 11. FFT matching between the template and the data image.

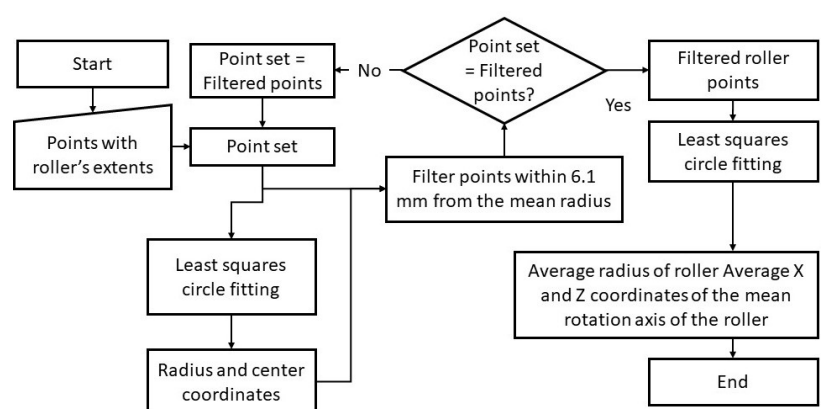

Figure 12. Flowchart to determine roller's points, average diameter, and the location of the mean rotation axis.

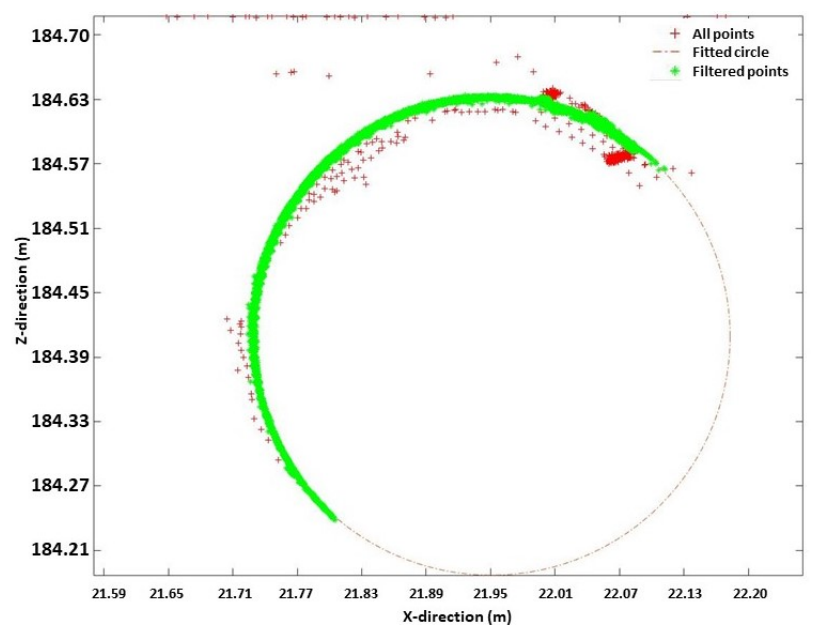

Figure 13. Laser points of one roller containing the roller points, outlier points, and the fitted circle.

different radii and centers' Z-coordinate. Thus, a two-stage filtering process was developed that examined the consistency in the radii and the centers of all the rollers to determine erroneously detected rollers. In the first stage, a moving average roller radius and Z-coordinate at each roller was calculated using the neighboring roller parameters. The differences between the computed and the average values were calculated and rollers outside certain confidence from the mean separation were con- sidered as outliers. By implementing the above process, erroneously detected rollers were excluded from further analysis. For each roller, its mean radius was added to the Z-coordinate of the roller's centers to determine the top elevation of the rollers' surface. In the second filtering stage, moving average filtering was implemented on the top elevation of the roller's surface to filter outliers. The analysis was performed using different moving average filter sizes and different confidences.

4.2.2 Determination of the individual roller's radius variation from the mean rotation axis: The steel slabs are primarily supported and transported over the middle length of the roller. Determining the radii variations over the length of the roller could provide essential information about its wear and tear. Therefore, radii variations of every roller with reference to the mean rotation axis were calculated at regular intervals to determine surface undulations. The roller points from the previous section were sliced in the Y-direction at every $25.4 \mathrm{~mm}$ ( 1 inch). A mean radius and its standard deviation were calculated with reference to the mean rotation axis for every slice of data. Figure 14 shows the radii variations and their standard deviations at each slice of the roller.

Class $A=S t$. Dev $\leq 1.58 \mathrm{~mm}$. Class $B=1.58 \mathrm{~mm}>$ St. Dev $\leq 3.18 \mathrm{~mm}$ Class $C=S t$ Dev $>3.18 \mathrm{~mm}$. Class $1=$ distance Class $A=S t . D e v \leq 1.58 m m$, Class $B=1.58 \mathrm{~mm}>\mathrm{St}$ Dev $\leq 3.18 \mathrm{~mm}$, Class $C=S \mathrm{St}$. Dev $>3$
from the mean radius $\leq 3.1 \mathrm{~mm}$ and Class $2=$ distance from the mean radius $>3.18 \mathrm{~mm}$

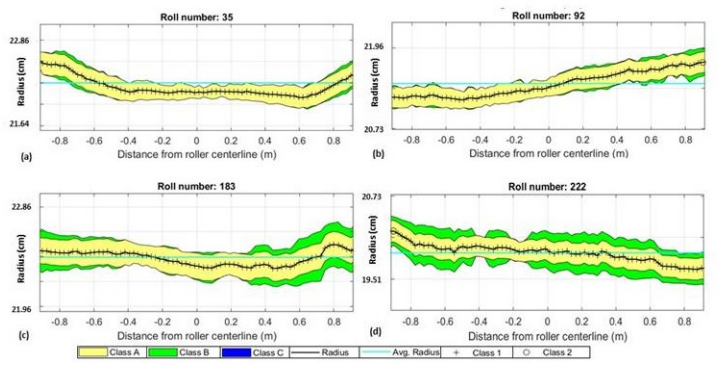

Figure 14. Radius calculated using every slice of the roller.

4.2.3 Determination of individual roller's actual (tilted) rotation axis: As described earlier, the rollers were susceptible to tilt. Tilt results in uneven wear and surface velocities. In this study, the roller's tilt was modeled by computing the radii at the ends of the roller as shown in Figure 15. Roller points within $0.3 \mathrm{~m}(1 \mathrm{ft})$ at both the ends of the roller were shortlisted to determine best-fit circles. The calculated radii and centers of these circles were assumed to be at the ends of the roller. The actual (tilted) rotation axis was created by connecting the centers at both ends of the roller. A positive tilt value represented that X' location was higher than X.

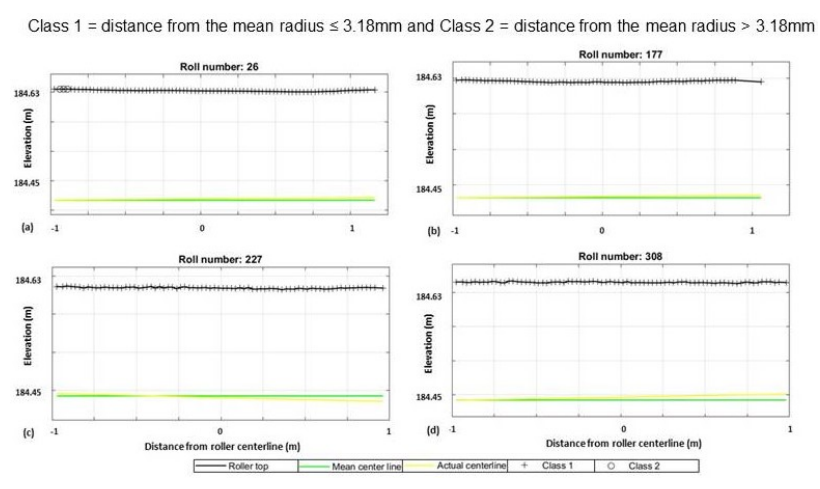

Figure 15. Mean and actual roller axis and the surface points.

4.2.4 Determination of the individual roller's radius variation from the actual (tilted) rotation axis: In this section, 
the roller surface variations with reference to the actual axis of rotation were determined. Roller points were divided into slices of $25.4 \mathrm{~m}$ width along its length to calculate the radii from the actual axis of rotation.

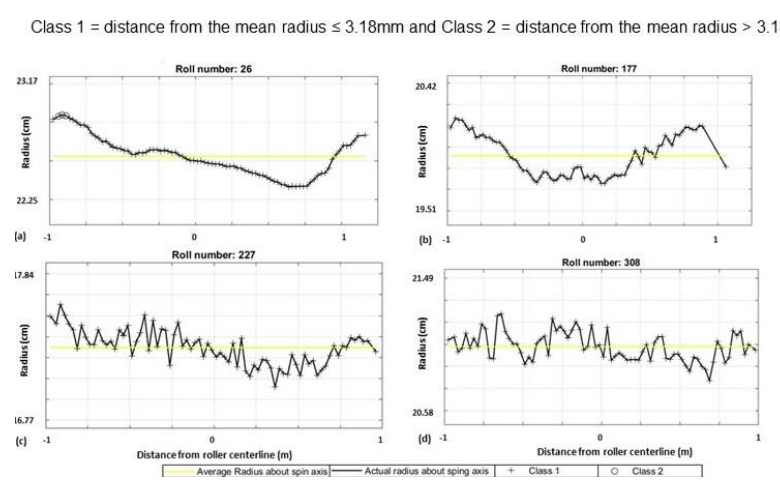

Figure 16. Mean and actual roller axis and the surface points.

\section{RESULTS AND DISCUSSIONS}

The above-presented methodologies were implemented on a dataset consisting of 372 rollers extending to a length of $350 \mathrm{~m}$. As it was not possible to include graphical results of all the rollers, salient graphics and tables have been shown to prove the efficacy of the methods.

\subsection{Locating rollers}

The original dataset covered 372 rollers. The points within a box placed around all the rollers were exported from Cyclone into MATLAB. These points were converted to voxels of dimensions $15.24 \mathrm{~mm}(0.05 \mathrm{ft}) \mathrm{x} 15.24 \mathrm{~mm}$ in the $\mathrm{X}$ and $\mathrm{Z}$ directions and extending over the entire width in the Y-direction. As explained in the 2D FFT image matching, the input image was created using the voxel dataset and the roller template image was created using a standard radius of $20.7 \mathrm{~cm}$. The entire length of the data was segmented into smaller sections consisting of approximately five rollers. A sufficient overlap was maintained between the sections so that no rollers at the edges were excluded. The rollers' locations identified in each region using the DFT were ordered in ascending X-coordinates and any duplicate locations were eliminated. Extents of each roller were defined from the matched location as explained in Figure 11(c) to segregate points belonging to each roller. Using the least-squares method, a circle was fitted iteratively to the segregated points to determine the roller's mean radius and mean center coordinates (mean axis of rotation).

\subsection{Assessment of roller location}

Using the 2D FFT, rollers were detected at 393 locations however, only 372 rollers existed in the data. Data for each roller was manually inspected to identify incorrect rollers. It was found that 366 rollers were correctly identified and modeled (see Figure 17), 6 rollers were correctly identified but incorrectly modeled (see Figure 18) and the remaining 21 were not roller locations (see Figure 19). Based on this inspection, the FFT matching successfully identified 98.3 percentage (366 out of 372) of rollers.

Figure 20(a), (b), and (c) show the Z-coordinate variations of roller's center, rollers' radii variations, and the rollers' top surface elevation variations, respectively. The 27 erroneously located or modeled rollers can be visually identified as spikes.
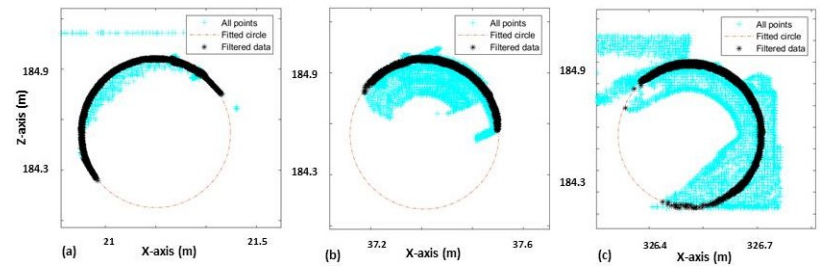

Figure 17. Sample locations with positive DFT matching and roller detection. Several outlier points can be seen that were filtered.

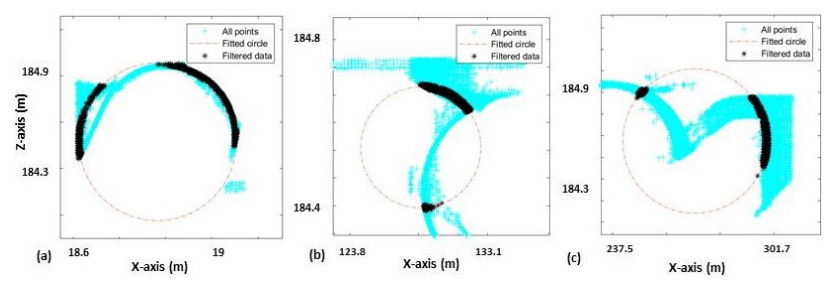

Figure 18. Sample locations where correct roller locations were identified but incorrect parameters were computed. (a) Outlier points on the left side of the roller. This resulted in a larger diameter radius and lower Z-coordinate of the center, (b) and (c) part of the roller selected resulting in incorrect parameters.

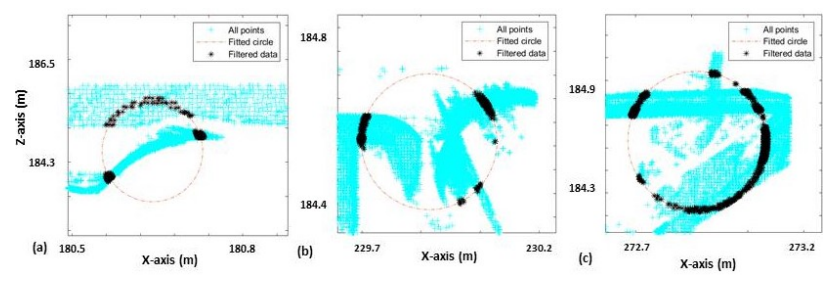

Figure 19. Sample identified locations where rollers were not present.

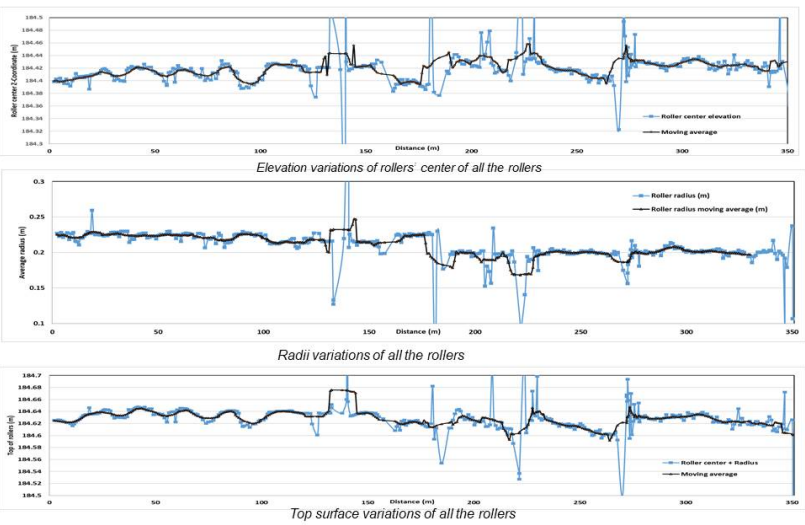

Figure 20. A plot showing the centerline of all rollers before filtering the erroneously located rollers.

\subsection{Filtering the data}

The erroneously located rollers were filtered in two steps. In the first step, the roller radii and Z-values were filtered using a moving average filter. Tests were conducted using two moving average filters to identify the outliers. The first moving filter 
used 5 locations (two on both sides and the location itself) and the second used 11 locations (five on both sides and the location itself) to compute the average values. Moving average values at the start and end of the line used fewer points. Figure 20(a) and (b) show the moving averages of the radii and $\mathrm{Z}$-values. The differences between the moving average and the calculated value at each roller were determined and points outside $68.8 \%$, $80 \%, 90 \%$, and $95 \%$ confidences about their mean differences were considered invalid. In the second step, the top elevations of every roller were computed by adding the roller radius to the center's Z-value. Once again, moving average values were computed and outliers were determined based on $68.8 \%, 80 \%$, $90 \%$, and $95 \%$ confidences. The efficacy of the above two steps filtering process was verified by visual inspection. Figure 21 summarizes the results. It can be noticed that by decreasing the confidence about the mean, a greater number of incorrect rollers were detected, but more correct rollers were also falsely flagged as outliers. Similarly, an increase in the size of the moving average filter showed better results. At $95 \%$ confidence using an 11-size moving average filter 18-locations out of 27-locations were detected as outliers. Nine locations were not detected, and 1 roller was falsely detected as an outlier. The total success rate was computed as a ratio of failed and incorrect locations to the total rollers in the data. In this case, $(366-9-1) / 366=97.3 \%$.

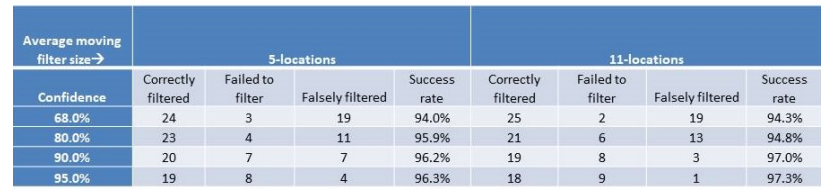

Figure 21. Filtering result at varying confidence intervals.

\subsection{Top surface variations after the two-step filtering}

Figure 22(a) and (b) shows the roller centerline and the radii variation plots after filtering. Continuing the roller's data analysis, desired pertinent measurements were also extracted for all the rollers. These results are discussed in the following sections. Filtered laser points for every roller were uniformly sliced to determine the average roller radii. The center of the circles was the mean rotation axis. Figure 14 illustrates surface variations with the reference to their mean axes of rotation for four sample rollers. The graphs show the areas of significant surface wear.

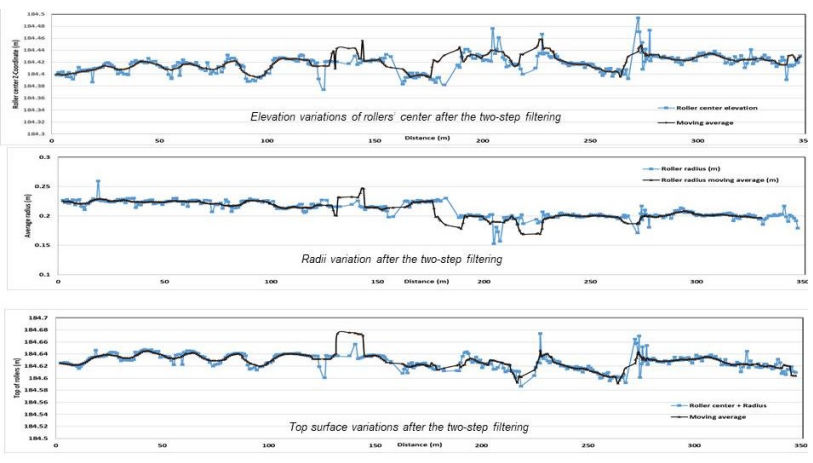

Figure 22. Centerline and radii variation after the two-stage filtering.

The actual (tilted) axis for each roller was subsequently determined by determining circles at both ends. A line connecting these circles was considered as the actual rotation axis. Figure 15 shows the mean and the actual axes of rollers' rotation.
The Class 1 and Class 2 points are placed at the radii distance from the mean axis of rotation. Figure 16 shows radii variations of rollers from the actual rotation axis. The radii variation highlights the surface wear for the rollers.

\section{CONCLUSION}

Point cloud consisting of 370 rollers and extending over $350 \mathrm{~m}$ was analyzed in this study. New methodologies were presented to locate and extract roller properties that are important in the steel industry. The primary results were visually inspected to determine the outlier. Two-stage moving average filters were used to identify the outliers. The results showed $97.3 \%$ success in identify rollers correctly using an 11-location moving average filter at $95 \%$ confidence. This study also extracted valuable information from the laser points data such as roller's erosion, tilt, and alignment parameters that can be useful to the steel industry to replace rollers. The results showed that the laser scanner can effectively map deformations in rollers.

During this investigation, several avenues were identified for future study including the use of image data were identified. At present, the entire process is automated with minimum human interpretation. Manual operations were only involved in the clipping the rollers from the entire steel mill dataset, the selection of a seed point, and defining the template roller size. The whole process can be improved to be an entirely automatic procedure, which allows for real-time data processing.

\section{REFERENCES}

Cabaleiro, M., Riveiro, B., Arias, P., Caamaño, J., 2015. Algorithm for beam deformation modeling from LiDAR data. Measurement, 76, 20-31.

Cabaleiro, M., Riveiro, B., Arias, P., Caamaño, J., 2016. Algorithm for the analysis of deformations and stresses due to torsion in a metal beam from LIDAR data. Structural Control and Health Monitoring, 23(7), 1032-1046.

Cabaleiro, M., Riveiro, B., Arias, P., Caamaño, J., Vilán, J., 2014. Automatic 3D modelling of metal frame connections from LiDAR data for structural engineering purposes. ISPRS Journal of Photogrammetry and Remote Sensing, 96, 47-56.

Chasmer, L., Hopkinson, C., Treitz, P., 2004. Assessing the three-dimensional frequency distribution of airborne and ground-based lidar data for red pine and mixed deciduous forest plots. Int Arch Photogramm Remote Sens Spat Inf Sci, 36(8), W2.

Deshpande, S. S., 2013. Improved floodplain delineation method using high-density LiDAR data. Computer-Aided Civil and Infrastructure Engineering, 28(1), 68-79.

Deshpande, S., Yilmaz, A., 2017. A semi-automated method to create a lidar-based hydro-flattened DEM. International journal of remote sensing, 38(5), 1365-1387.

Gézero, L., Antunes, C., 2019. Automated Three-Dimensional Linear Elements Extraction from Mobile LiDAR Point Clouds in Railway Environments. Infrastructures, 4(3), 46.

Liu, J.-K., Li, R., Deshpande, S., Niu, X., Shih, T.-Y. et al., 2009. Estimation of blufflines using topographic LiDAR data and orthoimages. Photogrammetric Engineering \& Remote Sensing, 75(1), 69-79. 
Popescu, S. C., Zhao, K., 2008. A voxel-based lidar method for estimating crown base height for deciduous and pine trees. Remote sensing of environment, 112(3), 767-781.

Puente, I., Akinci, B., González-Jorge, H., Díaz-Vilariño, L., Arias, P., 2016. A semi-automated method for extracting vertical clearance and cross sections in tunnels using mobile LiDAR data. Tunnelling and underground space technology, $59,48-54$.

Sánchez-Rodríguez, A., Soilán, M., Cabaleiro, M., Arias, P., 2019. Automated inspection of railway tunnels' power line using LiDAR point clouds. Remote Sensing, 11(21), 2567.

Selvaraj, S., Madhavan, M., 2018. Geometric imperfection measurements and validations on cold-formed steel channels using 3D noncontact laser scanner. Journal of Structural Engineering, 144(3), 04018010.

Smith, S. W. et al., 1997. The scientist and engineer's guide to digital signal processing. 14, California Technical Pub. San Diego.

Stein, D., Spindler, M., Kuper, J., Lauer, M., 2016. Rail detection using lidar sensors. International Journal of Sustainable Development and Planning, 11(1), 65-78.

Taheri Andani, M., Mohammed, A., Jain, A., Ahmadian, M., 2018. Application of LIDAR technology for rail surface monitoring and quality indexing. Proceedings of the Institution of Mechanical Engineers, Part F: Journal of Rail and Rapid Transit, 232(5), 1398-1406.

Yi, C., Lu, D., Xie, Q., Liu, S., Li, H., Wei, M., Wang, J., 2019. Hierarchical tunnel modeling from 3D raw LiDAR point cloud. Computer-Aided Design, 114, 143-154. 